\title{
La educación a pacientes y su corresponsabilidad como herramientas terapéuticas
}

\author{
Dora I. Molina*, Santiago Valencia-Uribe y Lina M. Agudelo-Rojas
}

Asociación IPS Médicos Internistas de Caldas, Manizales, Colombia

Recibido el 1 de febrero de 2016; aceptado el 6 de julio de 2016

Disponible en Internet el 12 de enero de 2017

\section{PALABRAS CLAVE \\ Factores de riesgo \\ cardiovascular; \\ Enfermedad \\ cardiovascular; \\ Tratamiento}

\section{KEYWORDS}

Cardiovascular risk

factors;

Cardiovascular

disease;

Treatment

\begin{abstract}
Resumen La enfermedad cardiovascular es la causa principal de muerte en el mundo. Con base en la importancia creciente de la corresponsabilidad de los pacientes en su cuidado, se ha intentado introducir la educación al paciente como herramienta terapéutica, de ahí la necesidad de emprender una revisión de la evidencia en la literatura médica que demuestre los resultados terapéuticos que se pueden obtener con la educación a pacientes, así como sus implicaciones y limitantes, particularmente en la salud cardiovascular de los individuos.

Se realizó una búsqueda sistemática en PubMed, a fin de elegir artículos desde 2005 hasta 2015, seleccionándose 40 manuscritos.

Se halló que la educación a pacientes, bajo diferentes modalidades, es una estrategia que ha adquirido gran acogida e importancia en el mundo en la atención integral de los pacientes, y ha demostrado beneficio en diversas entidades como factores de riesgo cardiovascular, insuficiencia cardiaca y enfermedad coronaria. Se encuentran también limitaciones serias al momento de diseñar los estudios, de modo que sus resultados se pueden ver subestimados.

Se concluye que la influencia que tiene la inclusión de la educación a pacientes dentro del manejo de estas enfermedades es significativa, y que por tanto esta debe ser un elemento central de los programas de manejo de la enfermedad cardiovascular y sus factores de riesgo. ( ) 2016 Sociedad Colombiana de Cardiología y Cirugía Cardiovascular. Publicado por Elsevier España, S.L.U. Este es un artículo Open Access bajo la licencia CC BY-NC-ND (http:// creativecommons.org/licenses/by-nc-nd/4.0/).
\end{abstract}

\section{Patient education and their co-responsibility as therapeutic tools}

Abstract Cardiovascular disease is the leading cause of death worldwide. An attempt to introduce patient education as a therapeutic tool has been made based on the growing importance of co-responsibility of patients in their care; in light of this a review of the evidence in medical literature showing the therapeutic results that can be obtained with patient education, as well as their implications and limits, especially regarding cardiovascular health of these individuals, needs to be conducted.

\footnotetext{
* Autor para correspondencia.

Correo electrónico: doraines56@gmail.com (D.I. Molina).
} 
A systematic search in PubMed was performed, choosing articles between years 2005 and 2015, selecting 40 papers.

It was found that patient education, under different forms, is an increasingly important and popular strategy worldwide in comprehensive care for patients, showing a benefit in multiple entities such as cardiovascular risk factors, cardiac insufficiency and coronary disease. Serious limitations are found in the design of the studies, so their results could be underestimated.

The study concludes that there is a significant influence on the inclusion of educating patients about the management of these conditions, and education must become a core element of the programmes for managing cardiovascular disease and its complications.

(c) 2016 Sociedad Colombiana de Cardiología y Cirugía Cardiovascular. Published by Elsevier España, S.L.U. This is an open access article under the CC BY-NC-ND license (http:// creativecommons.org/licenses/by-nc-nd/4.0/).

\section{Introducción}

La transición de una medicina tradicionalmente paternalista a una en la cual se tienen en cuenta la autonomía, las opiniones y decisiones del paciente en cuanto a su cuidado en salud, ubica la corresponsabilidad de los pacientes en un primer plano respecto a su manejo integral, pues es de importancia primaria que estos tomen decisiones educadas con base en información de calidad, respetando sus concepciones personales, culturales y religiosas.

Así, si se tiene en cuenta que gran parte del cuidado estará dado por el propio paciente, es ilusorio querer alcanzar una buena salud a toda costa, y en cambio, considerando el actual modelo de atención, buscar en consenso con este obtener una buena calidad de vida fundamentada en su libre albedrío. Por consiguiente, la educación para el paciente se vuelve un medio para lograr metas, que no necesariamente conducirá a una salud médica adecuada ${ }^{1}$.

Desde la publicación del reporte Lalonde en 1974, se resalta la importancia de la corresponsabilidad del paciente para buscar su salud y prevenir la enfermedad mediante cambios comportamentales como practicar ejercicio, adoptar una dieta saludable y evitar la exposición a sustancias peligrosas. Con la carta de Ottawa de 1986 y el desarrollo del modelo biopsicosocial en salud de la Organización Mundial de la Salud, se identifican al individuo y su contexto social como causas multifactoriales para su buena o mala condición de salud física y mental. Se crea también conciencia de los determinantes sociales de la salud, siendo necesaria la creación de programas para identificarlos e intervenirlos, tornándose así la promoción de la salud en una actividad fundamentalmente ligada a la educación ${ }^{2}$.

La enfermedad cardiovascular se conoce en la actualidad como la causa principal de muerte en el mundo. Se sabe, además, que la patología de origen cardiovascular, está estrechamente relacionada con diferentes factores de riesgo que, ampliamente se ha demostrado, representan un papel significativo en su etiopatogenia, entre ellos el tabaquismo, la hipertensión, la diabetes y la dislipidemia ${ }^{3,4}$. En la actualidad, a partir de los factores de riesgo conocidos y de su gran impacto en la génesis de las enfermedades cardiovasculares, dentro del enfoque intervencionista de la salud se ha tratado de introducir la educación al paciente.
Así pues, se hace necesario llevar a cabo una revisión de la evidencia en la literatura médica que demuestre los resultados terapéuticos que se pueden obtener con la educación a pacientes, así como sus implicaciones y limitantes, particularmente en la salud cardiovascular de los individuos.

\section{Métodos}

Se realizó una búsqueda sistemática en PubMed con los términos MeSH: ("patient education"'[Title]) AND ("patient education as topic' [MeSH Terms]) desde los años 2005 a 2015, únicamente en inglés y español. Se eligieron los trabajos de mayor peso científico como metaanálisis, revisiones sistemáticas, revisiones y estudios de calidad o citados con frecuencia.

\section{Resultados}

El buscador PubMed arrojó 745 resultados, de los cuales se escogieron los de mayor peso científico, mejor calidad y más citados. En total se seleccionan 40 manuscritos los cuales se referencian la mayoría en inglés. Se encontró abundante literatura producida por el área de enfermería, de gran importancia para el manejo integral y de educación a pacientes, si bien gran parte de los trabajos referenciados están constituidos por el área médica, y son sorprendentemente escasos.

\section{Discusión}

La educación a pacientes se ha convertido en esa forma directa a disposición del personal médico para transmitir conocimiento a los pacientes, a través de diferentes materiales de enseñanza como audiovisuales, medios impresos, sitios en la web, seguimiento asistencial telefónico, inclusión a grupos de pacientes con manejo multidisciplinario, entre otros ${ }^{4-6}$. No obstante, los estudios han demostrado lo limitados que son los programas educativos a pacientes, en cuyo caso, precisamente la falta de conciencia de los entes administrativos se ha convertido en una limitante para este pilar de manejo, y este hecho ha impedido que se revele su impacto en la población, aunándole a lo anterior los recursos 
escasos delegados para desarrollar los programas, el olvido de la estrategia por parte de los directivos institucionales y el deterioro de la relación médico-paciente que ataca la medicina, una disciplina deshumanizada por la tecnología que no ha permitido que la educación al paciente sea una propuesta aceptada. Respecto a este último punto en particular, en escasas ocasiones se ha tenido en cuenta en los planes de acción institucional y no se ha entendido que diversos estudios han mostrado una costo-efectividad significativa en el abordaje de los pacientes, siendo la inversión en tecnología médica descomunal para el manejo de pacientes crónicos, quienes posiblemente seguirán deteriorándose, sin considerar que la medida clave para reducir complicaciones de las mismas patologías, está en el conocimiento que tengan los pacientes de su enfermedad y en el tratamiento adecuado $^{7}$.

Con miras a contrarrestar lo anterior, el área de investigación científica en educación a pacientes y su corresponsabilidad, ha crecido exponencialmente en los últimos años; por ejemplo en Europa y Estados Unidos han surgido revistas dedicadas exclusivamente a la educación de pacientes, como "Patient education and counseling" de la European Association for Communication in Health Care en alianza con organizaciones americanas, en la que se abordan temas de gran impacto como educación en diabetes y alfabetismo en salud ${ }^{1,8}$.

\section{Desconocimiento crítico}

En el mundo el desconocimiento de los pacientes acerca de su salud cardiovascular es abrumador. En la población global abordada por el Prospective Urban Rural Epidemiological study (PURE), que posee población colombiana, se demostró que solo el $46,5 \%$ de los hipertensos estaban conscientes de su diagnóstico ${ }^{9}$.

En el estudio DAWN se probó que solo el $12 \%$ de los pacientes diabéticos llevaban a cabo medidas recomendadas de automanejo de la enfermedad ${ }^{10}$.

Europa ha encontrado en su población altos índices de desconocimiento de la enfermedad cardiovascular, así como carencia de conciencia frente a los malos hábitos de vida, relacionados significativamente con alteraciones cardio-metabólicas e inminente riesgo de eventos cardiocerebro-vasculares, predominando así un gran vacío en el conocimiento de factores de riesgo, prevalencias y formas de intervención de las diferentes patologías desde la condición de paciente ${ }^{11}$.

De otro lado, se ha demostrado en estudios norteamericanos que sólo $23 \%$ de personas han adoptado cambios de estilo de vida saludable y que además no estaban seguros de poder mantenerlos frente a diversos estresores o crisis de salud; un $12 \%$ afirmó seguir siendo sujetos pasivos al cuidado de la salud, un $29 \%$ refirió tener conocimiento sobre su enfermedad pero sin la confianza ni habilidad para lograr cambios basados en estos y los sujetos restantes no tenían conocimientos básicos ${ }^{12}$.

En el manejo de la información médica disponible para los pacientes influyen factores como los implícitos en la forma de presentación, factores ambientales y factores propios del paciente, siendo estos últimos los de mayor impacto tienen al momento de enfrentarse a la información ofrecida ${ }^{13}$. Está sustentado en la literatura que los pacientes con riesgo para tener un peor estado de salud son aquellos menos propensos a involucrarse en el cuidado de su propia condición; en este grupo se incluyen pacientes de escasos recursos, menos educados, con menor grado de alfabetismo en salud, ancianos y ya enfermos ${ }^{14}$. Estos grupos deberían ser prioritarios a la hora de educarles en salud y de comprometerles con su autocuidado.

De otro lado, se determinaron dos conceptos diferentes relacionados con la información médica: la lectura y la búsqueda ${ }^{13}$; dos actividades distintas asociadas con el uso de la misma, y adicionalmente se evidenció que la mayoría de los pacientes están interesados y propensos a leer la información pero no están dispuestos a hacer búsqueda activa; en cuanto a esto último, la población se ha acostumbrado a obtener la información en forma pasiva o consideran cuentan con suficiente o que es desgastante la búsqueda.

\section{Alfabetismo en salud}

Existe un factor fundamental para que esta estrategia cumpla su objetivo en la población, y es el grado de alfabetización de los pacientes, factor decisivo para que la misma pueda ser objeto de lectura y comprensión, así como propulsor de cambio, puesto que se ha demostrado que hay una relación directa entre bajo nivel de escolaridad y peor condición de salud, que conlleva gastos considerables para el sistema de salud ${ }^{13}$.

Como agravante, se ha comprobado que tanto el material impreso como el magnético dirigido a pacientes, excede su nivel y habilidad de lectura. Diversas organizaciones internacionales, incluida la National Institutes of Health y el Centers for Disease Control and Prevention, sugieren que en lo posible este material no debe exceder el sexto grado en compresión lectora; sin embargo, la mayoría del material, incluso el ofrecido por la National Library of Medicine, sobrepasa este nivel ${ }^{15,16}$, y por tanto se asume que toda persona que reciba información importante sobre su enfermedad, está en riesgo de no entenderla o malinterpretarla. Por esto, la Agency for Healthcare Research and Quality recomienda utilizar medidas precautorias al momento de diseñar material educativo en salud, afirmando que las organizaciones en salud tienen la responsabilidad de reducir el nivel educativo necesario para comprender tal material siguiendo unas pautas alternativas para el diseño de los documentos, que a la vez son útiles para la comunicación verbal con el paciente: presentar solo la información esencial, poner la información clave en primer lugar y usar medios alternativos ${ }^{17}$.

Dado el caso de que se recomiende material digital, se deben tener en cuenta los factores demostrados que se relacionen positivamente con este, como lo son haber nacido después de 1980, pertenecer al menos a clase socieconómica media, y tener un alto nivel de alfabetismo general ${ }^{15,18-20}$. En caso contrario y apreciando intuitivamente las características del paciente, se le debe ofrecer material impreso, a pesar de la creciente disponibilidad de la versión digital.

\section{Educación terapéutica}

Uno de los problemas críticos de gran crecimiento en nuestro sistema de salud es el poco tiempo que se deja al clínico 
para interactuar directamente con el paciente. Por ello se justifica la búsqueda de herramientas educativas no presenciales con el clínico, para así dedicar el tiempo que se tiene entre clínico y paciente a otras necesidades no satisfechas. Se debe emprender entonces una investigación adecuada a estas herramientas educativas y a su impacto ya que se pueden considerar en sí mismas armas terapéuticas.

Se esgrime el término de educación terapéutica como aquella que les ayuda a aprender, desarrollar y adaptar comportamientos que logran mejorar parámetros de salud, incluyendo biomarcadores y calidad de vida ${ }^{21}$.

La mayoría de los estudios sobre educación terapéutica indican que sus resultados han sido hacia la mejoría, mientras que otros han reportado efectos neutros y la menor cantidad han demostrado empeoramiento 22 .

Se ha estimado que solo el $33-50 \%$ de los pacientes con enfermedades crónicas tiene una adherencia completa a los medicamentos prescritos ${ }^{23}$, lo cual es tributario de intervención por la educación terapéutica.

Se ha sugerido, así mismo, que la educación a pacientes mejora las experiencias hospitalarias, al disminuir el dolor y la ansiedad ${ }^{24}$.

\section{Educación y factores de riesgo cardiovascular}

Datos de los estudios muestran que es plausible que la presencia de síntomas estimule a estos pacientes no sólo a buscar atención médica, sino información médica, permitiéndoles entender su condición y participar en su cuidado; pero lo que se ha podido deducir en cuanto a la hipertensión arterial y su manejo, es que su naturaleza asintomática se ha convertido en una de las principales razones para el incumplimiento o la interrupción del tratamiento, ya que estos pacientes, en comparación con aquellos que padecen patologías que comprometen su estilo de vida, consideran que no es fundamental el conocimiento de su patología; optan por aplicar la lectura, pero carecen de motivación o tal vez no ven necesaria la búsqueda activa de información escrita sobre hipertensión arterial, su manejo y complicaciones.

Para ilustrar la importancia de la educación, se encontró en un estudio reciente que con el solo hecho de que un material educativo enseñara las cifras tensionales normales, se obtuvo un $97 \%$ de aceptación de dicho material ${ }^{25}$.

En una revisión sistemática realizada por la colaboración Cochrane se encontró que la educación a pacientes logró disminuir la presión arterial ${ }^{26}$.

De igual forma, se destaca que hay un número creciente de pacientes que están interesados en llevar a cabo el automanejo de sus patologías, por ejemplo la hipertensión arterial, hecho de potencial utilidad para la automonitorización y el mayor control de las cifras tensionales ${ }^{25}$.

En lo concerniente al tratamiento de la diabetes mellitus, dado que hasta tres cuartos de estos pacientes fallece por enfermedad cardiovascular $^{27}$, cualquier medida que mejore resultados es bien recibida. A pesar de la carencia de evidencia de alta calidad, la educación en automanejo en diabetes como un proceso integral, es bien aceptada y es acogida ampliamente por diferentes organizaciones, incluida la American Diabetes Association.

Si bien se debe ofrecer desde el momento del diagnóstico, tiene muy baja tasa de adherencia por parte del paciente y su corresponsabilidad ${ }^{10}$. Se han intentado otros modelos educativos tales como el Diabetes Conversation Map program ${ }^{28}$, usado en más de 90 países, también acogido por la American Diabetes Association, que usa un abordaje de grupo altamente interactivo, empoderándoles de información así como apoyándoles para que ellos mismos hagan un plan de trabajo realista para alcanzar metas personales en salud ${ }^{29}$.

Otro estudio reciente con seguimiento a tres años en el que se evaluó una intervención educativa en pacientes con diabetes mellitus tipo 2 de novo, demostró que se dieron cambios en cuanto a creencias sobre la enfermedad, mas no hubo cambios sostenidos significativos en el estilo de vida y los parámetros biomédicos ${ }^{30}$.

Otra condición que requiere gran corresponsabilidad es el cuidado del pie diabético. Un metaanálisis de Cochrane demostró que en la mayoría de estos se mejoró el conocimiento y los cuidados autorreportados; sin embargo, solo en uno se demostró reducción en ulceración y amputación durante un año de seguimiento en pacientes de alto riesgo para ambas condiciones ${ }^{31}$.

En otros factores de riesgo cardiovasculares como la obesidad, se ha demostrado que la educación terapéutica tiene efectos benéficos en la pérdida de peso y el mantenimiento de esta ${ }^{32}$.

\section{Educación y pacientes cardiópatas}

Se determinó que gran parte de la literatura sobre educación terapéutica en cardiología es francesa. Como ejemplo, hay programas estandarizados en Francia que promueven la educación terapéutica en todos los centros de cardiología. Se ha logrado evidenciar que en pacientes con falla cardiaca crónica que se someten a educación terapéutica se disminuyen variables duras como la mortalidad a dos años, siendo aquella debida a cualquier causa del $17,3 \%$ en los pacientes educados vs. $31,0 \%$ en quienes no recibieron educación, bien cuando se incluyó la variable medicamentos y sin estos, confirmándose que la educación terapéutica es al menos parcialmente independiente de la medicación ${ }^{33}$.

Se ha reconocido el valor terapéutico de la educación en la mayoría de países industrializados ${ }^{33}$ y se ha encontrado que la presencia de comorbilidades dificulta la educación en pacientes con falla cardiaca ${ }^{34}$.

Una revisión sistemática que evaluó pacientes con enfermedad coronaria sugiere que las intervenciones educativas en este grupo, como se ha demostrado con otras patologías, aumentan el nivel de conocimiento y facilitan cambios comportamentales relacionados con el reconocimiento de síntomas y uso de medicamentos. Esta misma revisión señala que el educador más frecuente lo compone el personal de enfermería ${ }^{35}$.

En una revisión sistemática en nombre de la Sociedad Europea de Cardiología, con un total de 68.556 personas estudiadas, no se encontró una fuerte evidencia de que la educación redujera la morbilidad cardiaca definida como infarto subsecuente, revascularización u hospitalización y la mortalidad por todas las causas con un seguimiento de 18 meses en promedio. Sin embargo, se apreció mejoría en calidad de vida relacionada con la salud y menos uso de recursos en cuidado de la salud, aminorando por tanto 
los costos relacionados. Se encontró, además, que hay grupos poblacionales poco representados como las mujeres, los ancianos, personas no caucásicas, los revascularizados y los anginosos $^{36}$.

Así, recomiendan que todas las guías actuales para la prevención secundaria de enfermedad coronaria y rehabilitación cardiaca, contengan la recomendación de una intervención educativa integral que incluya ejercicio y apoyo psicológico.

En una revisión sistemática reciente se afirma que con el hecho de asegurar un acceso a programas de educación personalizados, integrales y basados en evidencia dirigidos a pacientes con enfermedad coronaria, se pueden optimizar los beneficios en relación con actividad física, cambios dietéticos y cese del tabaquismo ${ }^{35,37}$.

Los metaanálisis sugieren que en pacientes cardiacos las intervenciones dirigidas hacia un solo comportamiento son más exitosas ${ }^{37}$.

\section{Limitaciones}

Es una preocupación reiterativa en la mayoría de revisiones, que los métodos, específicamente la descripción relacionada con el tipo de intervención educativa, número de sesiones, participantes y duración, entre otros, no estén hechos de manera adecuada en la mayoría de los estudios analizados. Por tanto, la comparación de estudios y realización de metaanálisis se dificulta y no tiene el poder necesario, pues no hay certeza de qué intervenciones son exactamente las que se llevan a cabo; así, no hay datos suficientes para recomendar una clase de intervención educativa sobre otra. Por consiguiente, es imperativo que el investigador interesado en educación terapéutica y la corresponsabilidad de pacientes, preste especial atención a efectuar una descripción detallada de la intervención estudiada.

Cuando la intervención se estructura con base en indicadores precisos, con grupos control sin educación terapéutica, la educación muestra gran efectividad en las enfermedades crónicas ${ }^{22}$.

Otro factor que dificulta la investigación en educación pero que contribuye al arte de hacerlo, es el hecho de que ha sido una variable abordada desde dos puntos de vista, uno basado en la evidencia y otro en las humanidades. El primero se enfoca en demostrar las variables estadísticas cuantitativas relacionadas, similar por ejemplo a la administración de un medicamento, en tanto que el segundo analiza el contexto cultural y psicosocial. Es preciso, sin embargo, reconocer que la educación es una intervención compleja que no solo aplica a variables biomédicas ${ }^{38}$.

\section{Subestimación de resultados}

La eficacia de las intervenciones educativas puede verse subestimada por diferentes circunstancias: es casi imposible evitar darle cualquier información a un grupo control, pues esto no es ético y los pacientes por sí mismos se pueden informar por diferentes fuentes; la integralidad de las intervenciones educativas hace que cada variable y su magnitud sean difíciles de medir; puede ser improbable un proceso de aprendizaje aislado, ya que el medio educativo puede ser difícilmente controlado, pues los medios masivos como televisión, web y revistas pueden interactuar con el proveedor de salud ${ }^{22}$.

Se han estudiado otros métodos alternativos modernos con resultados hacia la mejoría, incluyendo el uso de páginas web como YouTube y Wikipedia ${ }^{39,40}$.

\section{Conclusiones}

La oferta de material educativo a la población, engloba completamente lo referente a promoción y prevención, propuesta terapéutica inclusiva que acoge tanto personas enfermas como no enfermas mediante información facilitada por los distintos medios audiovisuales y de forma escrita, con el fin de no limitar el acceso al material médico educativo, teniendo en cuenta los diferentes grados de alfabetización y posibles problemas de comprensión, atención o preferencias del público al cual va dirigido.

A la educación a pacientes, como componente indispensable en la terapéutica de las diferentes enfermedades, aún no se le ha dado por completo su verdadero valor en nuestro medio, de ahí que se espera que esta revisión tenga impacto en las instituciones colombianas.

Con base en los hallazgos, se recomienda su uso con el fin de crear conciencia en los pacientes acerca de la necesidad de conocer sobre su enfermedad, así como del papel que ellos mismos desempeñan en su cuidado, incrementando las tasas de efectividad de los tratamientos farmacológicos y disminuyendo complicaciones y aparición de comorbilidades relacionadas con su enfermedad base.

Es evidente que hacen falta estudios que continúen apoyando el uso y reflejen el impacto de la educación a pacientes en el área de la medicina. Por ahora es fundamental comprender su importancia como herramienta médica a favor de la población enferma y resaltar su utilidad como fuente de orientación y prevención en la población sana.

\section{Conflicto de intereses}

Los autores declaran no tener conflictos de interés.

\section{Bibliografía}

1. Hoving C, Visser A, Mullen PD, van den Borne B. A history of patient education by health professionals in Europe and North America: from authority to shared decision making education. Patient Educ Couns. 2010;78:275-81.

2. Ljungdalh AK, Møller JE. The social in medicine: social techniques in patient education. Health (London). 2012;16: 418-33.

3. Pearson TA, Blair SN, Daniels SR, Eckel RH, Fair JM, Fortmann SP, et al. AHA guidelines for primary prevention of cardiovascular disease and stroke: 2002 update. Circulation. 2002;106:388.

4. Harris M, Smith B, Veale A. Printed patient education interventions to facilitate shared management of chronic disease: a literature review. Int Med J. 2005;35:711-6.

5. Hulsmanemail RL, Steeleemail DJ. From patient education to health professions education: Introducing the new section on medical education. Patient Educ Couns. 2006;60:1-2.

6. Barlow J, Wright C, Sheasby J, Turner A, Hainsworth J. Self management approaches for people with chronic conditions: a review. Patient Educ Couns. 2002;48:177-87. 
7. Campbell M, Fitzpatrick R, Haines A, Kinmonth AL, Sandercock $P$, Spiegelhalter D, et al. Framework for design and evaluation of complex interventions to improve health. BMJ. 2000;321:694-6.

8. Anderson RM, Funnell MM. Patient empowerment: myths and misconceptions. Patient Educ Couns. 2010;79:277-82.

9. Chow CK, Teo KK, Rangarajan S, Islam S, Gupta R, Avezum A, PURE (Prospective Urban Rural Epidemiology) Study investigators. Prevalence, awareness, treatment, and control of hypertension in rural and urban communities in high-, middle-, and low-income countries. JAMA. 2013;310:959-68.

10. Funnell MM. The Diabetes Attitudes, Wishes, and Needs (DAWN) Study. Clin Diabetes. 2006;24:154-5.

11. Naudziunas A, Jankauskiene L, Kalinauskiene E, Pilvinis V. Implementation of the patient education about cardiovascular risk factors into a daily routine of the Cardiology Unit of the hospital. Prev Med. 2005;41:570-4.

12. Hibbard JH, Mahoney ER, Stock R, Tusler M. Do increases in patient activation result in improved self-management behaviors? Health Serv Res. 2007;42:1443-63.

13. Koo M, Krass I, Aslani P. Enhancing patient education about medicines: factors influencing reading and seeking of written medicine information. Faculty of Pharmacy, Bldg A15 The University of Sydney NSW. 2006. Australia.

14. Gruman J, Rovner MH, French ME, Jeffress D, Sofaer S, Shaller $D$, et al. From patient education to patient engagement: implications for the field of patient education. Patient Educ Couns. 2010;78:350-6.

15. Farnsworth M. Differences in perceived difficulty in print and online patient education materials. Perm J. 2014;18:45-50.

16. Stossel LM, Segar N, Gliatto P, Fallar R, Karani R. Readability of patient education materials available at the point of care. J Gen Intern Med. 2012;27:1165-70.

17. Papadakos CT, Papadakos J, Catton P, Houston P, McKernan P, Friedman JA. From theory to pamphlet: the $3 \mathrm{Ws}$ and an $\mathrm{H}$ process for the development of meaningful patient education resources. J Cancer Educ. 2014;29:304-10.

18. Prensky M. Digital natives, digital immigrants. On the Horizon. 2001;9:1-6.

19. Selber SA. Multiliteracies for a digital age. Carbondale, IL: SIU Press; 2004.

20. Hayles NK. How we think: digital media and contemporary technogenesis. Chicago, IL: University of Chicago Press; 2012.

21. Lagger G, Giordan A, Chambouleyron M, Lasserre Moutet A, Golay A. Therapeutic education. Part 2. Practice implication of 5 dimensional model. Médecine. 2008;4:269-73.

22. Lagger G, Pataky Z, Golay A. Efficacy of therapeutic patient education in chronic diseases and obesity. Patient Educ Couns. 2010;79:283-6.

23. Shoemaker SJ, Ramalho de Oliveira D, Alves M, Ekstrand $M$. The medication experience: preliminary evidence of its value for patient education and counseling on chronic medications. Patient Educ Couns. 2011;83:443-50.

24. Marcus C. Strategies for improving the quality of verbal patient and family education: a review of the literature and creation of the EDUCATE model. Health Psychol Behav Med. 2014;2:482-95.

25. Dawes MG, Kaczorowski J, Swanson G, Hickey J, Karwalajtys T. The effect of a patient education booklet and BP 'tracker' on knowledge about hypertension. A randomized controlled trial. Fam Pract. 2010;27:472-8.
26. Glynn LG, Murphy AW, Smith SM, Schroeder K, Fahey T. Interventions used to improve control of blood pressure in patients with hypertension. Cochrane Database Syst Rev. 2010. CD005182.

27. Tapp R, Shaw J, Zimmet P, eds. Complications of diabetes. In: International diabetes federation, ed. Diabetes atlas. 2 nd. ed. International Diabetes Federation, 2003.

28. Healthy Interactions. The U.S. Diabetes Conversation Map Program. Disponible en: http://healthyinteractions.com/ conversation-map-programs/conversation-mapexperience/current-programs/usdiabetes. [Acceso 24 Nov 2015].

29. Sperl-Hillen J, Beaton S, Fernandes O, Von Worley A, VazquezBenitez G, Parker E, et al. Comparative effectiveness of patient education methods for type 2 diabetes: a randomized controlled trial. Arch Intern Med. 2011;171:2001-10.

30. Khunti K, Gray LJ, Skinner T, Carey ME, Realf K, Dallosso H, et al. Effectiveness of a diabetes education and self management programme (DESMOND) for people with newly diagnosed type 2 diabetes mellitus: three year follow-up of a cluster randomised controlled trial in primary care. BMJ. 2012;344:e2333.

31. Dorresteijn JA, Kriegsman DM, Assendelft WJ, Valk GD. Patient education for preventing diabetic foot ulceration. Cochrane Database Syst Rev. 2014;12:CD001488.

32. Maldonato A, Piana N, Bloise D, Baldelli A. Optimizing patient education for people with obesity: possible use of the autobiographical approach. Patient Educ Couns. 2010;79: 287-90.

33. Juillière $Y$, Jourdain $P$, Suty-Selton $C$, Béard $T$, Berder V, Maître $B$, et al. Therapeutic patient education and all-cause mortality in patients with chronic heart failure: a propensity analysis. Int J Cardiol. 2013;168:388-95.

34. Dickson VV, Buck H, Riegel B. A qualitative meta-analysis of heart failure self-care practices among individuals with multiple comorbid conditions. J Card Fail. 2011;17:413-9.

35. Ghisi GL, Abdallah F, Grace SL, Thomas S, Oh P. A systematic review of patient education in cardiac patients: do they increase knowledge and promote health behavior change? Patient Educ Couns. 2014;95:160-74.

36. Brown JP, Clark AM, Dalal H, Welch K, Taylor RS. Effect of patient education in the management of coronary heart disease: a systematic review and meta-analysis of randomized controlled trials. Eur J Prev Cardiol. 2013;20:701-14.

37. Meng K, Seekatz B, Haug G, Mosler G, Schwaab B, Worringen U, et al. Evaluation of a standardized patient education program for inpatient cardiac rehabilitation: impact on illness knowledge and self-management behaviors up to 1 year. Health Educ Res. 2014;29:235-46.

38. Albano MG, Jourdain P, De Andrade V, Domenke A, Desnos M, d'Ivernois JF. Therapeutic patient education in heart failure: do studies provide sufficient information about the educational programme? Arch Cardiovasc Dis. 2014;107:328-39.

39. Stellefson M1, Chaney B, Ochipa K, Chaney D, Haider Z, Hanik $B$, et al. YouTube as a source of chronic obstructive pulmonary disease patient education: a social media content analysis. Chron Respir Dis. 2014;11:61-71.

40. Thomas GR, Eng L, de Wolff JF, Grover SC. An evaluation of Wikipedia as a resource for patient education in nephrology. Semin Dial. 2013;26:159-63. 